\title{
Erratum
}

\section{A Practical Synthesis of Highly Functionalized Fused 1,6-Dihydroimidazo-[1,2- a] imidazole-2,5-diones, Key Intermediates for LFA-1 Inhibitors}

Xiao-jun Wang,* Yibo Xu, Li Zhang, Dhileepkumar Krishnamurthy, Laurence Nummy, Vittorio Farina, Chris H. Senanayake Synlett, 2004, 2800.

In Scheme 1 the structure of compound 7 was incorrectly shown. The correct Scheme 1 is:

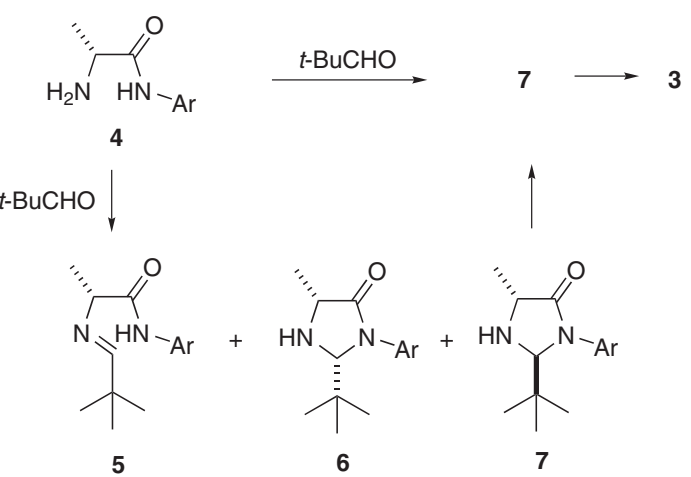

In Scheme 4 compound 9 was incorrectly represented. The correct Scheme 4 is:
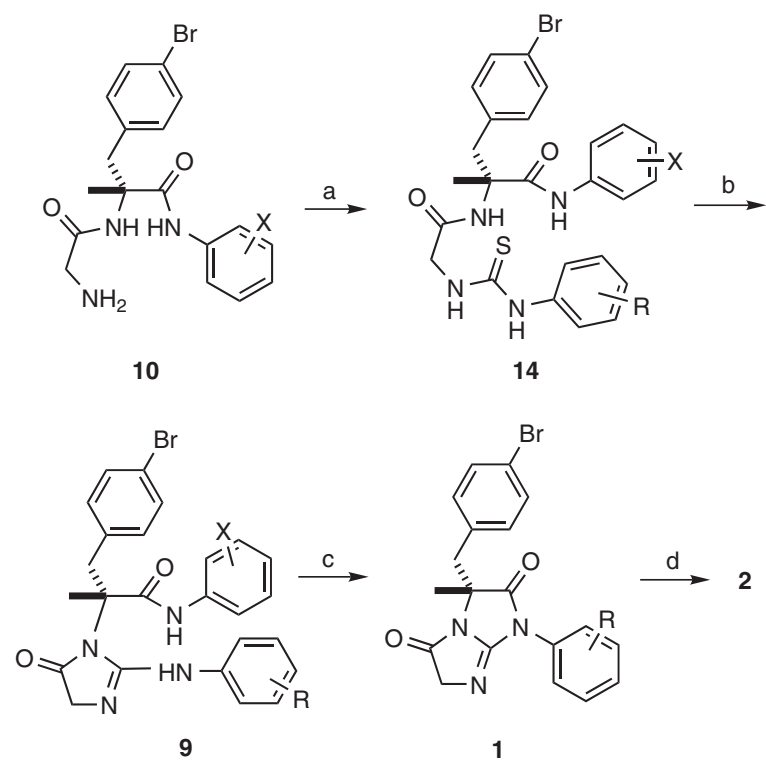

The authors and the editorial office apologize for these mistakes. 